ARTICLE

\title{
Hallmarks of the Mott-metal crossover in the hole-doped pseudospin-1/2 Mott insulator $\mathrm{Sr}_{2} \mathrm{IrO}_{4}$
}

Yue Cao ${ }^{1, \dagger}$, Qiang Wang ${ }^{1, \dagger}$, Justin A. Waugh ${ }^{1}$, Theodore J. Reber ${ }^{1, \dagger}$, Haoxiang $\mathrm{Li}^{1}$, Xiaoqing Zhou ${ }^{1}$, Stephen Parham¹, S.-R. Park ${ }^{1,2}$, Nicholas C. Plumb ${ }^{3}$, Eli Rotenberg ${ }^{4}$, Aaron Bostwick ${ }^{4}$, Jonathan D. Denlinger ${ }^{4}$, Tongfei $\mathrm{Qi}^{5}$, Michael A. Hermele ${ }^{1}$, Gang $\mathrm{Cao}^{5} \&$ Daniel S. Dessau ${ }^{1}$

The physics of doped Mott insulators remains controversial after decades of active research, hindered by the interplay among competing orders and fluctuations. It is thus highly desired to distinguish the intrinsic characters of the Mott-metal crossover from those of other origins. Here we investigate the evolution of electronic structure and dynamics of the hole-doped pseudospin-1/2 Mott insulator $\mathrm{Sr}_{2} \mid \mathrm{rO}_{4}$. The effective hole doping is achieved by replacing Ir with Rh atoms, with the chemical potential immediately jumping to or near the top of the lower Hubbard band. The doped iridates exhibit multiple iconic low-energy features previously observed in doped cuprates-pseudogaps, Fermi arcs and marginal-Fermi-liquidlike electronic scattering rates. We suggest these signatures are most likely an integral part of the material's proximity to the Mott state, rather than from many of the most claimed mechanisms, including preformed electron pairing, quantum criticality or density-wave formation.

\footnotetext{
${ }^{1}$ Department of Physics, University of Colorado, Boulder, Colorado 80309, USA. ${ }^{2}$ Department of Physics, Incheon National University, Incheon 22012, Korea. ${ }^{3}$ Swiss Light Source, Paul Scherrer Institut, Villigen PSI CH-5232, Switzerland. ${ }^{4}$ Advanced Light Source, Lawrence Berkeley National Laboratory, Berkeley, California 94720, USA. ${ }^{5}$ Department of Physics and Astronomy, Center for Advanced Materials, University of Kentucky, Lexington, Kentucky 40506, USA. $\dagger$ Present addresses: Condensed Matter Physics and Material Science Department, Brookhaven National Laboratory, Upton, New York 11973, USA (Y.C.) and (T.J.R.); Argonne National Laboratory, Lemont, Illinois 60439, USA (Q.W.). Correspondence and requests for materials should be addressed to Y.C. (email: ycao@colorado.edu) or to D.S.D. (email: Dessau@colorado.edu).
} 
F inding universal features in interacting electronic systems is a major theme in modern condensed matter research. By universality we refer to the low-energy properties that do not depend on the details of the interactions. For materials with relatively weak electron correlations, the low-energy excitations are well-described by the Fermi liquid theory. For doped Mott insulators where correlations are strong, pinpointing the hallmarks common to all Mott-metal crossovers ${ }^{1-3}$ has proven a formidable task. This could largely be attributed to the long candidate list of competing electronic orders, including longrange magnetic order and Fermi surface instabilities, among others, which yield a complex global doping-temperature phase diagram. For example, while the past three decades have witnessed tremendous progress in characterizing the phenomenology of the high-Tc cuprates, the interpretation of most of these experimental observations remains highly controversial. It has become essentially inseparable whether such exotic phenomena as pseudogaps and marginal Fermi liquid (MFL) scattering rates $^{2-5}$ arise from the metal-insulator transition, certain density-wave instabilities ${ }^{6}$ or are fluctuations of the superconductivity ${ }^{7}$. Moreover, the charge insulation in most known Mott insulators arises solely from the Coulomb repulsion. It would be highly desired to study new doped Mott insulators, especially those with a cleaner phase diagram (thus fewer competing orders) and a different mechanism that forbids electron double-occupancy.

$\mathrm{Sr}_{2} \mathrm{IrO}_{4}$ has attracted much interest recently as a new family of Mott insulator ${ }^{8,9}$, with the potential of realizing novel phases of matter ${ }^{10}$ and achieving higher superconducting $\mathrm{T}_{\mathrm{C}}{ }^{11}$. The Ir-O planes are similar to the $\mathrm{Cu}-\mathrm{O}$ planes in cuprates, with the Ir atoms sitting at the centre of the Ir-O octahedra, except for the $22^{\circ} \mathrm{Ir}$-O-Ir bond angle (Fig. 1a). There are five $\mathrm{t}_{2 \mathrm{~g}}$ electrons per Ir. The Ir $t_{2 g}$ level splits into the $J_{3 / 2}$ doublet (filled with four electrons) and the $J_{1 / 2}$ singlet as a result of the strong spin-orbit coupling. The half-filled $J_{1 / 2}$ further splits into lower (filled with 1 electron) and upper (empty) bands, with this splitting general believed to be due to the Coulomb repulsion, which is why these bands are considered upper and lower Hubbard bands (UHB and LHB, see Fig. 1c $)^{9}$. The insulating behaviour in $\mathrm{Sr}_{2} \mathrm{IrO}_{4}$ derives from the coupling of strong spin-orbit interaction with Coulomb repulsions, which is drastically different from that in cuprates. However, the $\mathrm{Sr}_{2} \mathrm{IrO}_{4}$ parent compound, just like undoped cuprates, is antiferromagnetically ordered ${ }^{12}$. Driving $\mathrm{Sr}_{2} \mathrm{IrO}_{4}$ towards metallicity thus provides a unique opportunity to investigate the universal features of the Mott-metal crossover.

Different approaches of doping $\mathrm{Sr}_{2} \mathrm{IrO}_{4}$ and related compounds have been found ${ }^{13-17}$, and of special interest is the $\mathrm{Sr}_{2} \mathrm{Ir}_{1-x} \mathrm{Rh}_{x} \mathrm{O}_{4}$ series. With as little as $4 \% \mathrm{Rh}$ substitution, the normalized resistivity drops by $10^{4}$. The long-range magnetic order decays more slowly and still survives with a $\mathrm{T}_{\mathrm{N}} \sim 17 \mathrm{~K}$ for $15 \% \mathrm{Rh}$ (see Supplementary Fig. 1). Rh is directly above Ir in the periodic table, so is expected to be isovalent. It has been proposed that the metallicity in the Rh-doped iridates comes from the reduced spin-orbit coupling of $\mathrm{Rh}$ (due to the smaller atomic number) which then leads to the reduced splitting of the $J_{3 / 2}$ and $J_{1 / 2}$ bands $^{15,16}$, as well as the formation of in-gap states ${ }^{16}$ (Fig. 1e). As we will show below, the $\mathrm{Rh}$ atoms in fact act as effective hole dopants to $\mathrm{Sr}_{2} \mathrm{IrO}_{4}$ (Fig. 1d). The chemical potential moves to the top of the LHB, without major band renormalizations.

So far, no superconductivity has yet been reported in these Rh-doped compounds, which is different from doped cuprates. The absence of superconductivity reduces possible competing orders and makes the Rh-doped iridates a cleaner system to study-the long-range canted antiferromagnetism (AF) is the only confirmed order in the system. In the following, we show in the effectively hole-doped $\mathrm{Sr}_{2} \mathrm{IrO}_{4}$ the emergence of pseudogaps, Fermi arcs and marginal-Fermi-liquid-like electronic scattering rates, features first reported and/or most famous in doped cuprate Mott insulators. It appears these iridates resemble cuprates not only structurally but also in terms of electronic structures.

\section{Results}

Effective hole doping via Rh substitution. Angle-resolved photoemission spectroscopy (ARPES) proves an invaluable tool for directly observing both electronic structure and low-energy electron dynamics in doped Mott insulators ${ }^{3}$ and bears implication for electronic order, including density-wave instabilities. We performed ARPES on single crystals of $\mathrm{Rh}$-doped $\mathrm{Sr}_{2} \mathrm{IrO}_{4}$. Details of the sample growth are listed in a

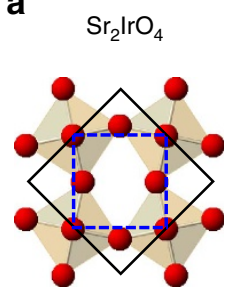

b

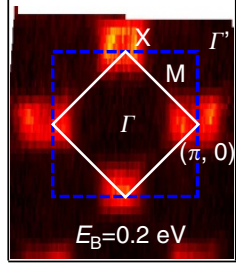

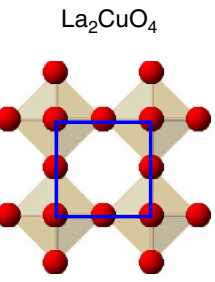

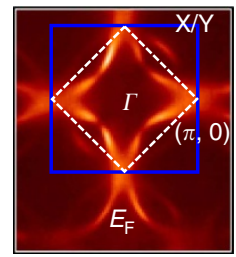

C

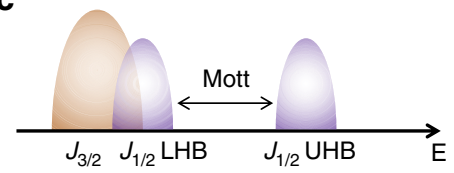

d

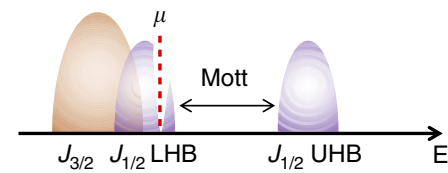

e (Inconsistent with ARPES)

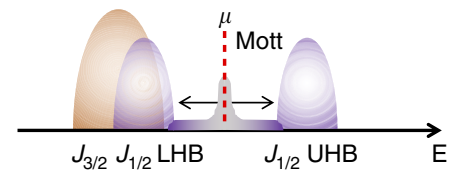

Figure 1 | $\mathbf{S r}_{2} \mathrm{IrO}_{\mathbf{4}}$ as a Mott insulator on the square lattice. (a) The real-space unit cells of $\mathrm{Sr}_{2} \mathrm{IrO}_{4}$ and $\mathrm{La}_{2} \mathrm{CuO}_{4}$. (b) The $\mathrm{k}$-space unit cells of the same, with matching colour scaling and with near- $E_{F}$ ARPES spectral weight. Ignoring the $22^{\circ} \mathrm{Ir}-\mathrm{O}$ twists gives the blue cells in real and $\mathrm{k}$-space, and corresponds to the regular unit cell of $\mathrm{La}_{2} \mathrm{CuO}_{4}$. Including these twists in $\mathrm{Sr}_{2} \mathrm{IrO}_{4}$ (black, (a)) back-folds the k-space cell in k-space (white), similar to the AF order in the parent cuprates. (c) The formation of the Mott gap in $\mathrm{Sr}_{2} \mathrm{IrO}_{4}$ as a result of the spin-orbit coupling and Coulomb interaction. (d,e) Possible schematics of chemical potential evolution with Rh doping. 
the Methods section and Supplementary Note 1; and the ARPES experiment setups are presented in the Methods section and Supplementary Note 2 . Due to the $\sqrt{2} \times \sqrt{2}$ lattice reconstruction, the Brillion zone (BZ) of $\mathrm{Sr}_{2} \mathrm{IrO}_{4}$ and its Rh-doped compounds is reduced in half, similar to the formation of the antiferromagnetic (AF) $\mathrm{BZ}$ in the parent cuprates. We show the constant energy surfaces of $\mathrm{Sr}_{2} \mathrm{IrO}_{4}$ (Fig. 1b, left panel) and as a comparison, of $\mathrm{Pb}$-doped $\mathrm{Bi}_{2} \mathrm{Sr}_{2} \mathrm{CaCu}_{2} \mathrm{O}_{8+\delta}$ (Fig. 1b, right panel). The folded and 'original' $\mathrm{BZs}$ for the iridates are marked in white solid and blue dashed lines, respectively. The 'original' BZ corresponds to one Ir-O plaquette in real space, as shown by the blue dashed line in the left panel in Fig. 1a. As we will show later in the paper, the close-to- $E_{\mathrm{F}}$ features of the doped iridates are best captured not by the folded BZ, but instead by the 'original' blue BZ. To avoid confusion, we use $(\pi, 0)$ to mark the $\mathrm{X}$ point in the $\mathrm{BZ}$ as defined in ref. 9, and use $\Gamma$ ' to denote $(\pi, \pi)$, which is the $\Gamma$ point in the second folded BZ. In Fig. 2a,b, we show the constant energy surfaces as a function of binding energy for $\mathrm{Sr}_{2} \mathrm{IrO}_{4}$ and $\mathrm{Sr}_{2} \mathrm{Ir}_{1-x} \mathrm{Rh}_{x} \mathrm{O}_{4}$ with $x=15 \%$ at $\mathrm{T}=50 \mathrm{~K}$. While there is no Fermi surface for the parent compound, there are states at $E_{\mathrm{F}}$ in the $x=15 \%$ iridate, corresponding to enhanced conductivity in the Ir-O plane. The constant energy surface of the $x=15 \%$ compound is quite similar to that of the parent, except that it is shifted in binding energy by $\sim 200 \mathrm{meV}$. To identify the Fermi surface topology, we plot the ARPES spectrum along $\Gamma^{\prime}-(\pi, 0)$ for both samples (Fig. 2c; along the yellow lines in Fig. 2a,b). There is a hole-pocket centred at $(\pi, 0)$, which comes from the $J_{1 / 2}$ $\mathrm{LHB}^{9,18}$. The top of the valence band is $\sim 170 \mathrm{meV}$ below $E_{\mathrm{F}}$ in the parent compound, and is above $E_{\mathrm{F}}$ for the $x=15 \%$ sample. Indeed, both the $J_{3 / 2}$ band (white dashed line in Fig. $2 c$ ) and $J_{1 / 2}$ LHB (green dashed line) ${ }^{19,20}$ are shifted by $\sim 200 \mathrm{meV}$. It appears that rather than a reduced splitting of the $J_{3 / 2}$ and $J_{1 / 2}$ bands, $\mathrm{Sr}_{2} \mathrm{IrO}_{4}$ is hole doped with $\mathrm{Rh}$ substitution. Note, that while the $\mathrm{Rh}$-doped compound displays strong spectral weight extending towards $(\pi / 2, \pi / 2)$, the $\mathrm{M}$ point near $E_{\mathrm{F}}$, the band dispersion at the $\mathrm{M}$ point lies below $E_{\mathrm{F}}$ (see Supplementary Fig. 2 and Supplementary Note 3 ). Therefore, the 'Fermi surface' is made up only of the states encircling $\mathrm{X}$ or $(\pi, 0)$, that is, it encompasses holes.

We determined the chemical potential shift quantitatively from the valence bands, as shown in Fig. 3a. With the increase of Rh concentration, the chemical potential is pushed deeper into the
$J_{1 / 2} \mathrm{LHB}$, confirming that $\mathrm{Rh}$ acts as an effective hole dopant. We extrapolate the chemical potential shift at finite $\mathrm{Rh}$ densities and derive an $\sim 170 \mathrm{meV}$ intercept in the zero doping limit. Note, the top of the valence band in the parent compound locates at $(\pi, 0)$ and is around $170 \mathrm{meV}$ below $E_{\mathrm{F}}$, from both ARPES $^{18}$ and scanning-tunneling ${ }^{21}$ experiments. Thus on effective hole doping the chemical potential immediately jumps to the edge of the LHB (Fig. 1d), as opposed to competing models (Fig. 1e) ${ }^{16,22}$ where new quasiparticle-like states emerge in the middle of the Hubbard gap. The doping schematic in Fig. 1d also agrees with the recent optical conductivity measurements ${ }^{16}$, while the interpretation in ref. 16 (Fig. 1e) could not explain the ARPES measured band structure (see Supplementary Note 4).

The effective hole doping is quite plausible when considering the simple atomic model depicted in Fig. $3 \mathrm{~b}$. Rh atoms have smaller spin-orbit coupling than Ir, leading to the smaller

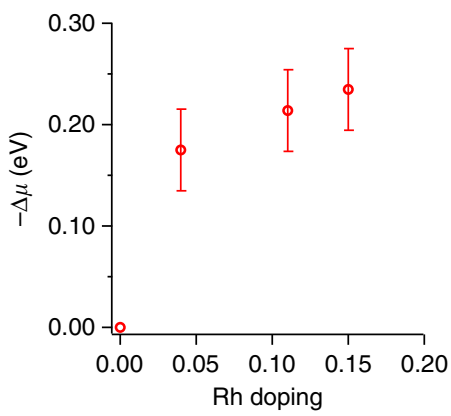

b

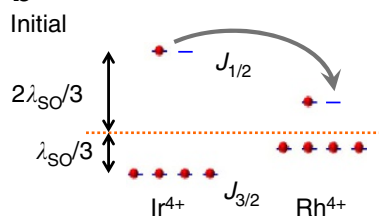

Final

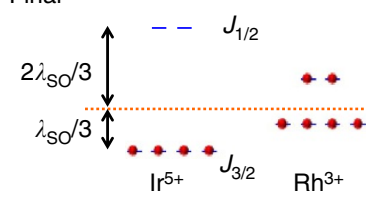

Figure 3 | $\mathbf{~ R h}$ atoms act as hole dopants in $\mathbf{S r}_{\mathbf{2}} \mathbf{I r O} \mathbf{4}_{\mathbf{4}}$ (a) Chemical potential shift versus $\mathrm{Rh}$ concentration. The chemical potential shift is measured from the shift of both $J_{1 / 2} \mathrm{LHB}$ and $J_{3 / 2}$ bands. The error bar for each Rh concentration is the half-width of the positive peaks of the secondderivatives of EDCs, averaged over a few high-symmetry k points.

(b) Simple atomic picture of hole doping, ignoring band effects and Mott splitting. With a roughly similar average energy for both Ir and Rh sites, the smaller on-site spin-orbit splitting on the Rh sites lowers the $J_{1 / 2}$ energy relative to that of the host Ir sites. This causes an electron transfer to Rh, that is, hole doping of the Ir lattice. a

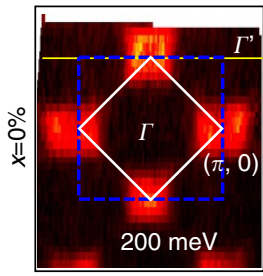

b

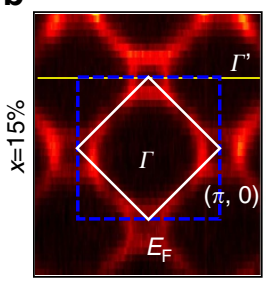

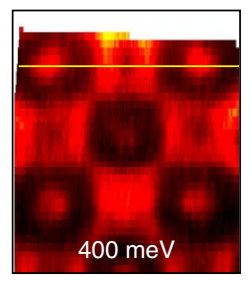

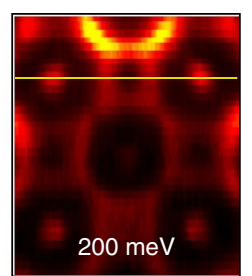

c
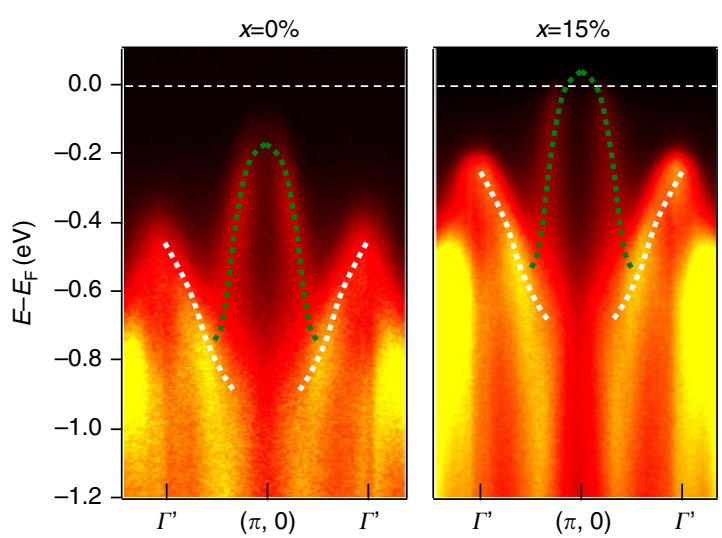

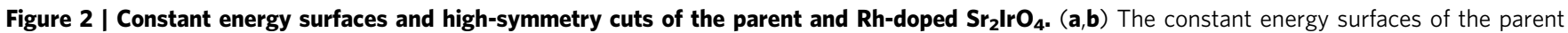
(a) and $x=15 \%$ Rh-substituted (b) $\mathrm{Sr}_{2} \mathrm{IrO}_{4}$. The numbers are binding energies relative to $E_{\mathrm{F}}$. The solid white/dashed blue lines are the folded/original $\mathrm{BZs}$. (c) ARPES energy-momentum intensity plots along $\Gamma^{\prime}-(\pi, 0)-\Gamma^{\prime}$ (yellow lines in panels $(\mathbf{a}, \mathbf{b})$ ). The dashed green and white lines through the data guide the eye for the $J_{1 / 2}$ and $J_{3 / 2}$ bands, respectively. 
splitting between the $J_{3 / 2}$ and $J_{1 / 2}$ states. Assuming the average energy of all six $t_{2 g}$ states is similar for both Rh and Ir, the empty $J_{1 / 2}$ state of Rh would then have a lower energy than that of Ir. Thus a $J_{1 / 2}$ electron from a neighbouring Ir atom will fill the $J_{1 / 2}$ state on the Rh site, leaving behind a hole on the Ir site, as well as a filled and immobile $\mathrm{Rh}^{3+}$ site. Of course this is a simplistic model that neither takes into account the finite bandwidth of the effective J states nor the Coulomb repulsion U. Recent X-ray absorption experiments at the $\mathrm{Rh}_{\mathrm{L}}$-edge have confirmed $\mathrm{Rh}$ indeed has a valence of $3+$ in these compounds ${ }^{23}$.

Fermi arcs and pseudogaps. Hereafter, we focus on the lowenergy electronic dynamics of these hole-doped compounds in search for 'universal' features during the Mott-metal crossover. In Fig. 4a we show the Fermi surface topology for the $x=15 \%$ sample at $50 \mathrm{~K}$. The segments of the Fermi surface centred at $\Gamma\left(\Gamma^{\prime}\right)$ and equivalent k locations are highlighted with solid yellow (blue) lines, and labelled FS1 and FS2, respectively. Energydistribution curves (EDCs) from many different $\mathrm{k}$ points on the Fermi surface are plotted in Fig. 4c. There is a marked difference between the EDCs from FS1 and FS2-those from FS2 are generally pushed away from $E_{\mathrm{F}}$. We refer to this spectral weight suppression as a 'pseudogap' and use the standard 'midpoint of leading edge' method ${ }^{24,25}$ to quantify it, by fitting the EDCs to a shifted, broadened leading edge (see Supplementary Note 5), with the amount of shift (defined as the gap size) giving the $50 \%$ point of the leading edge. Note that in contrast to the case of a BCS-like gap that works well for superconductivity or a standard charge or spin density-wave gap, this shifted edge does not have a pile-up of spectral weight beyond the gap edge, like many other pseudogaps $s^{4,25}$, that is, it does not enforce spectral weight conservation on the opening of the gap. This absence of quasiparticle peaks in the doped iridates is one of the signatures of their non-Fermi liquid nature. We assign the gap size $\Delta_{1}$ and $\Delta_{2}$ from the leading edge fitting to FS1 and FS2, respectively. For $x=15 \%, \Delta_{1}$ as defined by this leading edge method vanishes, and FS1 can be considered a 'regular' piece of Fermi surface (albeit without quasiparticle peaks), while EDCs from FS2 show a partial depletion of near- $E_{\mathrm{F}}$ spectral weight. As FS1 is only topologically connected to the 'gapped' FS2, we describe the FS1 as a Fermi surface 'arc' and FS2 as 'pseudogapped'.

We track how $\Delta_{1}$ and $\Delta_{2}$ evolve with reduced Rh concentration, as the material gets closer to the Mott insulator. At $x=4 \%$ (Fig. 4b), both $\Delta_{1}$ and $\Delta_{2}$ are finite, indicating that both FS1 and FS2 are pseudogapped, with $\Delta_{1} \sim 3 \mathrm{meV}$ and an increased $\Delta_{2} \sim 38 \mathrm{meV}$. In Fig. $4 \mathrm{~d}$ we plot the doping dependence of $\Delta_{1}$ and $\Delta_{2}$. For $x=4 \% \sim<11 \%$, the entire Fermi surface is pseudogapped, which resembles the deeply underdoped cuprates ${ }^{26,27}$. We mark the presence of both pseudogaps as suppressed spectral weight near $E_{\mathrm{F}}$ (the 'notch') in Fig. 1d. The pseudogaps observed here are not to be mistaken for the matrix element effect as these gaps happen over a very narrow-binding energy range that is essentially identical for different photon energies (see Supplementary Note 6).

The pseudogap phase in correlated electron systems is often considered a symmetry-broken phase of matter. Thus the origin of the pseudogap could be reflected in its k-space symmetry, as well as its thermal evolution. For Rh concentration $4-15 \%, \Delta_{1}$ $\left(\Delta_{2}\right)$ is roughly independent of $\mathbf{k}$ along the Fermi surface segment FS1 (FS2). In this sense, the pseudogaps in the non-superconducting $\mathrm{Rh}$-doped iridates are clearly different from the near-nodal prepairing pseudogap in the near-optimal cuprates $^{28}$, where pseudogaps follow the superconducting paring symmetry. a

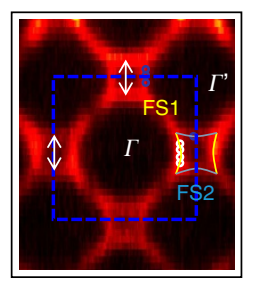

d

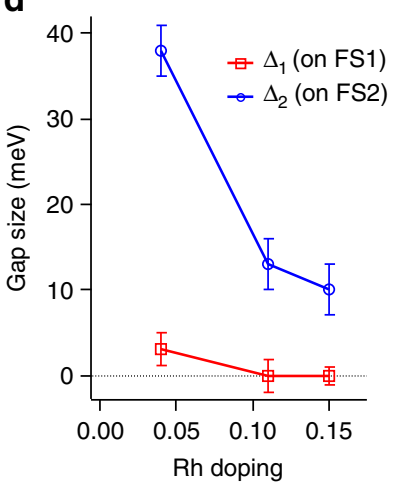

b

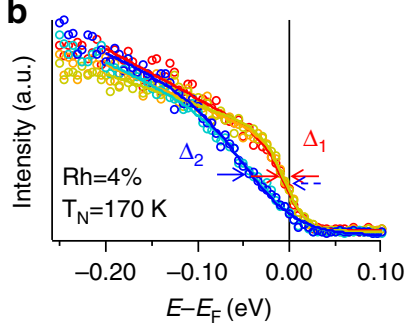

c
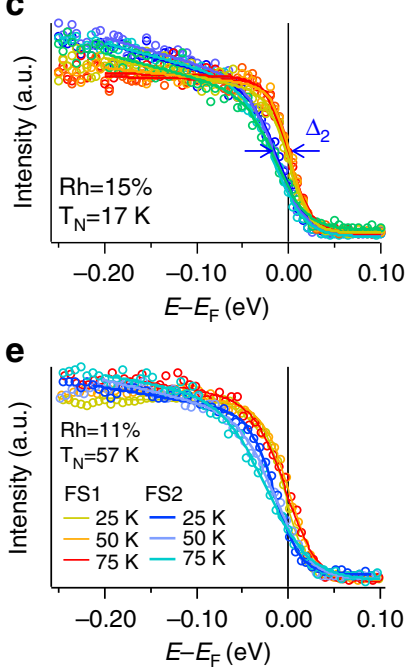

Figure 4 | Fermi surface segments and pseudogaps in hole-doped

$\mathbf{S r}_{\mathbf{2}} \mathbf{I} \mathbf{O}_{\mathbf{4}}$. All the data were taken at $50 \mathrm{~K}$ unless otherwise noted. (a) Fermi surface spectral weight of the $x=15 \%$ sample, with a hole-like Fermi pocket centred around the $(\pi, 0)$ point of the unfolded (blue dashed) BZ. The FS pocket is separated into segments FS1 (yellow) and FS2 (blue), with FS1 facing $\Gamma$ and FS2 facing $\Gamma^{\prime}$. Q vectors (white arrows) are possible densitywave nesting vectors. $(\mathbf{b}, \mathbf{c})$ EDCs from multiple locations along the FS1 and FS2 segments (yellow and blue, respectively) taken from the $x=4 \%$ and $x=15 \%$ samples. Locations of the individual EDCs are marked by the open coloured circles in $\mathbf{a}$. The leading edges of most EDCs do not reach $E_{F}$, that is, they are gapped or pseudogapped. Gap sizes extracted using the 'midpoint of leading edge' method, are shown in $\mathbf{b}, \mathbf{c}$ and compiled in $\mathbf{d}$, with $\Delta_{1}$ labelling the gaps from FS1 and $\Delta_{2}$ the gaps from FS2. The uncertainties of $\Delta_{1}$ and $\Delta_{2}$ are defined as the standard deviation of the fitted gap sizes of individual EDCs from the averaged value. (e) EDCs from FS1 (dashed) and FS2 (solid) showing minimal temperature dependence across the magnetic phase transition of the $x=11 \%$ sample.

Figure $4 \mathrm{e}$ shows the temperature dependence of EDCs at $\mathrm{k}_{\mathrm{F}}$ from FS1 and FS2 for the $x=11 \%$ sample, with the temperature range straddling the AF ordering temperature $T_{N}=57 \mathrm{~K}$. Within the error bar no obvious changes with temperature are observed, indicating that the pseudogap is not directly related to the longrange canted AF order. This is further confirmed in the $x=15 \%$ sample (Fig. 4c), where $\Delta_{2}$ is finite at $50 \mathrm{~K}$ and persists to at least $200 \mathrm{~K}$ (see Supplementary Fig. 3 and Supplementary Note 5), above $T_{N}=17 \mathrm{~K}$. This observation suggests the pseudogap is not tied to the long-range magnetic order, and it is likely the pseudogap phase persists down to the zero-temperature quantum ground state in these hole-doped iridates.

Another commonly considered origin of pseudogaps is the density-wave instabilities in the form of Fermi surface nesting, as has been discussed in the manganites ${ }^{25}$ and cuprates ${ }^{6,29}$. In the case of iridates, it is temping to draw nesting vectors such as the white arrows (Fig. 4a) between FS2's with the same gap size $\Delta_{2}$. However, the same ordering vector Q also connects FS1's, yet 
with a much smaller gap $\Delta_{1}$. The Fermi surface nesting scenario does not explain the preference for a larger gap along FS2 than along FS1. It appears the pseudogap in iridates is inconsistent with many influential explanations for the antinodal pseudogap 25,29 in manganites and near optimally doped cuprates though it may be more connected with the more-recently observed nodal pseudogap in heavily underdoped cuprates $^{26,27}$.

Marginal-Fermi-liquid like single-electron scattering rate. The non-Fermi liquid nature of these doped iridates is not only reflected in the absence of quasiparticle peaks along the EDC (Fig. $4 \mathrm{~b}, \mathrm{c}$ ), but also in the single-electron scattering rate. In the Fermi liquid theory, the quasiparticle scattering rate grows linearly with the binding energy (and temperature) squared. For up to $15 \% \mathrm{Rh}$ substitution, as shown in Fig. $5 \mathrm{~b}$ (with raw data shown in Fig. 5a) the scattering rates increase roughly linearly with binding energy-a signature of the MFL ${ }^{5}$. We could rule out this linear scattering rate as from the Rh-Ir substitution, and further details are shown in the Supplementary Fig. 4 and Supplementary Note 7. Moreover, as shown in Fig. 5c, there is a linear relation between the resistivity and the sample temperature, as highlighted by the black dashed line. Here we have ignored the upturn of the resistivity at low temperatures that is likely due to a localization effect, as has also been observed in most of the underdoped cuprates $^{30}$. The linear MFL scattering rate is one of the most iconic features of the cuprates and other correlated materials such as the ruthenates ${ }^{31}$ and has been attributed to a wide range of ideas including quantum critical fluctuations ${ }^{5}$ and the break-up of quasparticles 32 .

\section{Discussion}

The simultaneous appearance of Fermi arcs, pseudogaps and the MFL state in both iridates and cuprates is unlikely to be coincidental. We note recent measurements of electron-doped iridates using a range of spectroscopies, including ARPES $33-35$, scanning-tunneling microscope ${ }^{36}$ and resonant inelastic X-ray scattering $^{37}$, also with features similar to those in cuprates. Our study and these new findings suggest there is a wide resemblance between doped iridates and cuprates in terms of band structures, electron dynamics and collective excitations, and on both the hole and electron-doped sides of the phase diagram. As cuprate is a canonical doped Mott insulator, and the most thoroughly studied one so far, our knowledge of a doped Mott insulator is to a great extend bounded by the cuprate literature. In this sense, the aforementioned hallmarks are most likely intrinsic and universal, rather than material specific, during the Mott-metal crossover.

We have ruled out prepairing and Fermi surface nesting as accountable for the pseudogaps in the iridates. Also there is no known quantum critical point in the doped iridates. We could also rule out long-range AF and structural distortions: both the pseudogap and the MFL survive above $T_{N}$ in the iridates, and static structural distortions are less likely to be related to the electron dynamics.

The precise definition of a pseudogap is in itself debated. From an experimental perspective, a widely accepted working criterion is well-articulated by Timusk and Statt ${ }^{4}$ in the mid-1990s in the study of cuprates: a 'pseudogap' is a partial gap, or more general, an incomplete suppression of spectral weight, that occurs only on some regions of the Fermi surface. Experimental features observed in, for example, ARPES, scanning-tunneling microscope, optical conductivity, all stem out of this seemingly naive (and more general) working definition. Admittedly, this definition does not point to the nature of pseudogaps. There are some cases where the origin of pseudogaps are understood theoretically to various degrees. For example, pseudogaps from preformed Cooper pairs usually have a momentum dependence characteristic of the pairing symmetry $3,28,38$, with relatively well-defined Bogoliubov quasiparticles. On the other hand, pseudogaps due to Fermi surface nesting features 'hotspots' on the Fermi surface connected by the charge/orbital/magnetic ordering vector ${ }^{25,29}$, which could be detected in other techniques.

Notably, even in these cases, not all facets of the pseudogap of interest, for example, its evolution against temperature, follows known theories. For example, the prepairing pseudogap ${ }^{28}$ in the optimally doped cuprates does evolve continuously into a 'real' hardened gap with decreasing temperature into the superconducting regime. However, as temperature rises, the prepairing pseudogap has been reported ${ }^{38,39}$ to be killed due to density of states filling-in rather than a reduction in gap size with increasing temperature, unlike standard Bogoliubov quasiparticles, reflecting their non-Fermi liquid nature. In other cases, a 'real' hardened gap and a defined phase transition may never develop when lowering the temperature towards absolute
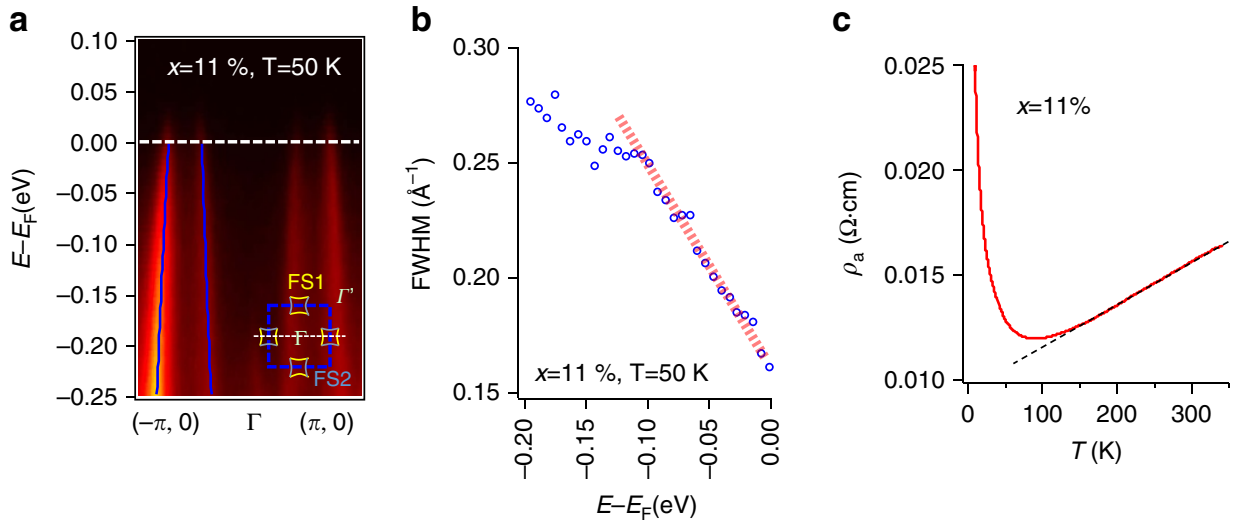

Figure 5 | Marginal-Fermi-liquid-like single-electron scattering rates. (a) The energy-momentum intensity plot along $(-\pi, 0)-\Gamma-(\pi, 0)$ for an $x=11 \%$ sample at $T=50 \mathrm{~K}$, passing through four pieces of FS1 Fermi surface. The peak centroids obtained from double-Lorentzian fittings to the momentum-distribution curves (MDCs) are marked with blue lines. The inset shows where the cut in the main figure is taken from the BZ. (b) The fullwidth-half-maximum (FWHM) from the double-Lorentzian fitting is plotted versus the binding energy, showing a linear 'MFL' scattering rate. (c) Resistivity versus temperature for the same sample showing a linear dependence at intermediate temperatures. The black dashed line is a guide to the eye. 
zero from the pseudogap phase. For example, in the p-type cuprates with a hole doping of 5-6\%, the pseudogap temperature is generally above $200 \mathrm{~K}$, and the ground state is considered by many a spin-glass without any defined phase transition ${ }^{3,40}$. There is no known clear boundary between the pseudogap phase and the spin-glass in this regime.

As we pointed out at the forefront of this paper, here we define pseudogaps following the definition from Timusk and Statt ${ }^{4}$. We have shown the pseudogaps and Fermi arcs in these iridates are distinctive from the prepairing and charge/orbital/ spin ordered ones. The survival of pseudogaps to these seemingly high temperatures, compared with the Neel temperature, is a reflection of the Mott nature of iridatessomething that is very relevant considering the recent debates over whether $\mathrm{Sr}_{2} \mathrm{IrO}_{4}$ is a Mott insulator or a Slater insulator ${ }^{41,42}$. The main disagreement between the two types of insulators is the role of short-range AF correlations. The Slater insulator is a mean-field concept built on the formation of long-range order-in the present case the long-range AF order. The short-range AF correlations that underlie the long-range AF order is ignored, or at best considered as a higher-order fluctuation. It is expected that the gap in a Slater insulator will diminish with decreasing long-range magnetic order, trending to zero as the phase transition is approached. Experimentally, there is no clear change in the band structure for both the parent compound $^{18,43}$ and the doped iridates (this work) across the onset of long-range magnetism. This indicates the long-range magnetic order is not necessary for the formation of the gap and $\mathrm{Sr}_{2} \mathrm{IrO}_{4}$ is a Mott insulator.

Our observation of hallmarks during the Mott-metal crossover due to their proximity to the Mott state is in contrast with many of the prevailing views in the literature, but similar to those suggested in a few recent theoretical works ${ }^{44,45}$. More specifically, the smooth crossover from the Mott insulator $\mathrm{Sr}_{2} \mathrm{IrO}_{4}$ to the hole-doped 'bad metal', suggests the essential role of short-range correlations in the low-energy electron dynamics. It is interesting to point out the short-range AF correlations may be responsible for many of the hallmarks during the Mott-metal crossover, and in regions of the phase diagram where long-range AF order is long gone. For example, short-range AF correlations could indicate a widespread distribution of AF interaction strengths and quantum incoherence, giving rise to the lack of quasiparticle peaks and less well-defined gapping near the Fermi level-a possibility for iridates and even cuprates, among other doped Mott insulators.

The relation between the AF energy scale and the pseudogap temperatures is far from clear, with the strengths of the AF interactions converted to $>1,000 \mathrm{~K}$ in iridates (and cuprates). In the latter the pseudogap temperature $\mathrm{T}^{\star}$ neither tracks the AF phase nor the superconducting dome. As for iridates, at this stage, we are not yet sure whether the pseudogap closes or could be filled due to thermal effects, at a higher temperature $T^{\star}$. Our data here would suggest most likely that there is a gradual filling-in of the density of states with rising temperature, while the actual $\mathrm{T}^{*}$ (if it can be well-defined) is yet to be determined (see Supplementary Note 5 for details). Interestingly the community does not have a consensus whether the pseudogap actually closes or is filled in the underdoped cuprates. Regardless, this type of study, in which we closely consider the similarities and differences in the behaviour of the iridates and cuprates as they evolve away from the Mott insulator is a powerful tool to delve deeply into the nature of the doped Mott insulating state.

\section{Methods}

Sample preparation. All the ARPES, transport and magnetization data were taken from bulk $\mathrm{Sr}_{2} \mathrm{Ir}_{1-x} \mathrm{Rh}_{x} \mathrm{O}_{4}$ samples. Single crystals were grown from off-stoichiometric quantities of $\mathrm{SrCl}_{2}, \mathrm{SrCO}_{3}, \mathrm{IrO}_{2}$ and $\mathrm{RhO}_{2}$ using self-flux techniques.
ARPES measurements. The ARPES experiments were performed at the PGM-A end station at the Synchrotron Radiation Center of University of WisconsinMadison, the Beamline 4.0.3 and 7.0.1 ARPES end stations at the Advanced Light Source, Lawrence Berkeley National Laboratory and the Surface and Interface Science beamline of the Swiss Light Source at the Paul Scherrer Institut. The samples were cleaved in situ with vacuum better than $5 \times 10^{-11}$ Torr. The band structure and low-energy spectra near the Fermi level were taken with $h v=77,80$, and $90 \mathrm{eV}$, with an energy resolution $\sim 25 \mathrm{meV}$, which is sharp on the scale of the principle spectral features.

\section{References}

1. Imada, M., Fujimori, A. \& Tokura, Y. Metal-insulator transitions. Rev. Mod. Phys. 70, 1039-1263 (1998).

2. Lee, P. A., Nagaosa, N. \& Wen, X.-G. Doping a Mott insulator: physics of hightemperature superconductivity. Rev. Mod. Phys. 78, 17-85 (2006).

3. Damascelli, A., Hussain, Z. \& Shen, Z.-X. Angle-resolved photoemission studies of the cuprate superconductors. Rev. Mod. Phys. 75, 473-541 (2003).

4. Timusk, T. \& Statt, B. The pseudogap in high-temperature superconductors: an experimental survey. Rep. Prog. Phys. 62, 61-122 (1999).

5. Varma, C. M. Pseudogap phase and the quantum-critical point in copper-oxide metals. Phys. Rev. Lett. 83, 3538-3541 (1999).

6. Comin, R. et al. Charge order driven by Fermi-arc instability in $\mathrm{Bi}_{2} \mathrm{Sr}_{2-x} \mathrm{La}_{x} \mathrm{CuO}_{6+\delta}$. Science 343, 390-392 (2014).

7. Anderson, P. W. The resonating valence bond state in $\mathrm{La}_{2} \mathrm{CuO}_{4}$ and superconductivity. Science 235, 1196-1198 (1987).

8. Kim, B. J. et al. Phase-sensitive observation of a spin-orbital Mott state in $\mathrm{Sr}_{2} \mathrm{IrO}_{4}$. Science 323, 1329-1332 (2009).

9. Kim, B. J. et al. Novel $\mathrm{J}_{\mathrm{eff}}=1 / 2$ Mott state induced by relativistic spin-orbit coupling in $\mathrm{Sr}_{2} \mathrm{IrO}_{4}$. Phys. Rev. Lett. 101, 076402 (2008).

10. Jackeli, G. \& Khaliullin, G. Mott insulators in the strong spin-orbit coupling limit: from Heisenberg to a quantum compass and Kitaev models. Phys. Rev. Lett. 102, 017205 (2009).

11. Wang, F. \& Senthil, T. Twisted Hubbard model for $\mathrm{Sr}_{2} \mathrm{IrO}_{4}$ : magnetism and possible high temperature superconductivity. Phys. Rev. Lett. 106, 136402 (2011).

12. Ye, F. et al. Magnetic and crystal structures of $\mathrm{Sr}_{2} \mathrm{IrO}_{4}$ : a neutron diffraction study. Phys. Rev. B 87, 140406 (2013).

13. Ge, M. et al. Lattice-driven magnetoresistivity and metal-insulator transition in single-layered iridates. Phys. Rev. B 84, 100402 (2011).

14. Qi, T. F. et al. Electron doped $\mathrm{Sr}_{2} \mathrm{IrO}_{4-\delta}(0 \leq \delta \leq 0.04)$ : evolution of a disordered $\mathrm{J}_{\mathrm{eff}}=1 / 2$ Mott insulator into an exotic metallic state. J. Appl. Phys 109, 07D906 (2011).

15. Qi, T. F. et al. Spin-orbit tuned metal-insulator transitions in single-crystal $\mathrm{Sr}_{2} \mathrm{Ir}_{1-x} \mathrm{Rh}_{x} \mathrm{O}_{4}(0 \leq x \leq 1)$. Phys. Rev. B 86, 125105 (2012).

16. Lee, J. S. et al. Insulator-metal transition driven by change of doping and spin-orbit interaction in $\mathrm{Sr}_{2} \mathrm{IrO}_{4}$. Phys. Rev. B 85, 035101 (2012).

17. Dhital, C. et al. Carrier localization and electronic phase separation in a doped spin-orbit-driven Mott phase in Sr3(Ir1-xRux)2O7. Nat. Commun. 5, 3377 (2014).

18. Wang, Q. et al. Dimensionality-controlled Mott transition and correlation effects in single-layer and bilayer perovskite iridates. Phys. Rev. B 87, 245109 (2013).

19. Zhang, H., Haule, K. \& Vanderbilt, D. Effective $J=1 / 2$ insulating state in Ruddlesden-Popper iridates: an LDA + DMFT study. Phys. Rev. Lett. 111, 246402 (2013).

20. Martins, C., Aichhorn, M., Vaugier, L. \& Biermann, S. Reduced effective spin-orbital degeneracy and spin-orbital ordering in paramagnetic transitionmetal oxides: $\mathrm{Sr}_{2} \mathrm{IrO}_{4}$ versus $\mathrm{Sr}_{2} \mathrm{RhO}_{4}$. Phys. Rev. Lett. 107, 266404 (2011).

21. Dai, J.-X. et al. Local density of states study of a spin-orbit-coupling induced Mott insulator $\mathrm{Sr}_{2} \mathrm{IrO}_{4}$. Phys. Rev. B 90, 041102(R) (2014).

22. Allen, J. W. et al. Resonant photoemission study of $\mathrm{Nd}_{2-x} \mathrm{Ce}_{x} \mathrm{CuO}_{4-y}$ : nature of electronic states near the Fermi level. Phys. Rev. Lett. 64, 595-598 (1990).

23. Clancy, J. P. et al. Dilute magnetism and spin-orbital percolation effects in $\mathrm{Sr}_{2} \mathrm{Ir}_{1-x} \mathrm{Rh}_{x} \mathrm{O}_{4}$. Phys. Rev. B 89, 054409 (2014).

24. Shen, Z.-X. et al. Anomalously large gap anisotropy in the a-b plane of $\mathrm{Bi}_{2} \mathrm{Sr}_{2} \mathrm{CaCu}_{2} \mathrm{O}_{8+\delta}$. Phys. Rev. Lett. 70, 1553-1556 (1993).

25. Chuang, Y.-D. et al. Fermi surface nesting and nanoscale fluctuating charge/orbital ordering in colossal magnetoresistive oxides. Science 292, 1509-1513 (2001).

26. Razzoli, E. et al. Evolution from a nodeless gap to $\mathrm{dx}^{2}-\mathrm{y}^{2}$-wave in underdoped $\mathrm{La}_{2}-{ }_{x} \mathrm{Sr}_{x} \mathrm{CuO}_{4}$. Phys. Rev. Lett. 110, 047004 (2013).

27. Vishik, I. M. et al. Phase competition in trisected superconducting dome. Proc. Natl Acad. Sci. USA 109, 18332-18337 (2012).

28. Reber, T. J. et al. The origin and non-quasiparticle nature of Fermi arcs in $\mathrm{Bi}_{2} \mathrm{Sr}_{2} \mathrm{CaCu}_{2} \mathrm{O}_{8+\delta}$. Nat. Phys. 8, 606-610 (2012).

29. He, R.-H. et al. From a single-band metal to a high-temperature superconductor via two thermal phase transitions. Science 331, 1579-1583 (2011).

30. Ando, Y. et al. Mobility of the doped holes and the antiferromagnetic correlations in underdoped high- $\mathrm{T}_{\mathrm{C}}$ cuprates. Phys. Rev. Lett. 87, 017001 (2001). 
31. Grigera, S. A. et al. Magnetic field-tuned quantum criticality in the metallic ruthenate $\mathrm{Sr}_{3} \mathrm{Ru}_{2} \mathrm{O}_{7}$. Science 294, 329-332 (2001).

32. Anderson, P. W. The 'strange metal' is a projected Fermi liquid with edge singularities. Nat. Phys. 2, 626-630 (2006).

33. Kim, Y. K. et al. Fermi arcs in a doped pseudospin-1/2 Heisenberg antiferromagnet. Science 345, 187-190 (2014).

34. Kim, Y. K. et al. Observation of a d-wave gap in electron-doped $\mathrm{Sr}_{2} \mathrm{IrO}_{4}$. Nat. Phys. 12, 37-41 (2016).

35. de la Torre, A. et al. Collapse of the Mott gap and emergence of a nodal liquid in lightly doped $\mathrm{Sr}_{2} \mathrm{IrO}_{4}$. Phys. Rev. Lett. 115, 176402 (2015).

36. Yan, Y. J. et al. Electron-doped $\mathrm{Sr}_{2} \mathrm{IrO}_{4}$ : an analogue of hole-doped cuprate superconductors demonstrated by scanning tunneling microscopy. Phys. Rev. X 5, 041018 (2015).

37. Liu, X.-R. et al. Anisotropic softening of magnetic excitations in lightly electron doped $\mathrm{Sr}_{2} \mathrm{IrO}_{4}$. Preprint at http://arxiv.org/abs/1601.02172 (2016).

38. Reber, T. J. et al. Prepairing and the 'filling' gap in the cuprates from the tomographic density of states. Phys. Rev. B 87, 060506 (2013).

39. Kondo, T. et al. Disentangling Cooper-pair formation above the transition temperature from the pseudogap state in the cuprates. Nat. Phys. 7, 21-25 (2011).

40. Norman, M. R., Pines, D. \& Kallin, C. The pseudogap: friend or foe of high Tc? Adv. Phys. 54, 715-733 (2005).

41. Hsieh, D. et al. Observation of a metal-to-insulator transition with both MottHubbard and Slater characteristics in $\mathrm{Sr}_{2} \mathrm{IrO}_{4}$ from time-resolved photocarrier dynamics. Phys. Rev. B 86, 035128 (2012).

42. Arita, R. et al. Ab initio studies on the interplay between spin-orbit interaction and Coulomb correlation in $\mathrm{Sr}_{2} \mathrm{IrO}_{4}$ and $\mathrm{Ba}_{2} \mathrm{IrO}_{4}$. Phys. Rev. Lett. 108, 086403 (2012).

43. Moser, S. et al. The electronic structure of the high-symmetry perovskite iridate $\mathrm{Ba}_{2} \mathrm{IrO}_{4}$. New J. Phys. 16, 013008 (2014).

44. Civelli, M. et al. Dynamical breakup of the Fermi surface in a doped Mott insulator. Phys. Rev. Lett. 95, 106402 (2005).

45. Kyung, B. et al. Pseudogap induced by short-range spin correlations in a doped Mott insulator. Phys. Rev. B 73, 165114 (2006).

\section{Acknowledgements}

Y.C. and D.S.D. acknowledge J.-X. Dai, K. McElroy, D. Reznik, X.-W. Zhang, A. Zunger, G. Arnold, D. Haskel, J. P. Clancy and Y.-J. Kim for insights and discussions. Y.C. also thanks Y.-D. Chuang, M. Bissen, M. Severson for their help in setting up the experiment. D.S.D. acknowledges support from the U.S. Department of Energy (DOE), Office of
Science, Basic Energy Sciences (BES) under Award \# DE-FG0203ER4606. G.C. acknowledges support by NSF via grant DMR 1265162. M.A.H. was supported by the U.S. Department of Energy (DOE), Office of Science, Basic Energy Sciences (BES) under Award \# DE-FG02-10ER46686. The ARPES data were collected in part from the Synchrotron Radiation Center, University of Wisconsin-Madison, which was initially supported by the National Science Foundation under Award No. DMR-0537588, and later primarily funded by the University of Wisconsin-Madison with supplemental support from facility Users and the University of Wisconsin-Milwaukee. The ARPES data were also taken from the Advanced Light Source and the Swiss Light Source. The former is supported by the Director, Office of Science, Office of Basic Energy Sciences, of the U.S. Department of Energy under Contract No. DE-AC02-05CH11231.

\section{Author contributions}

Y.C., Q.W., J.A.W., T.J.R., H.X.L., X.Q.Z., S.P., S.-R.P. and N.C.P. performed the ARPES experiments with help from E.R., A.B. and J.D.D. T.F.Q. and G.C. grew and characterized the samples. M.A.H. developed the explanation for the effective hole doping picture. Y.C analysed the ARPES data. Y.C. and D.S.D wrote the majority of the paper with inputs from all authors. D.S.D. directed the project.

\section{Additional information}

Supplementary Information accompanies this paper at http://www.nature.com/ naturecommunications

Competing financial interests: The authors declare no competing financial interests

Reprints and permission information is available online at http://npg.nature.com/ reprintsandpermissions/

How to cite this article: Cao, Y. et al. Hallmarks of the Mott-metal crossover in the hole-doped pseudospin-1/2 Mott insulator $\mathrm{Sr}_{2} \mathrm{IrO}_{4}$. Nat. Commun. 7:11367 doi: $10.1038 /$ ncomms11367 (2016).

(c) (i) This work is licensed under a Creative Commons Attribution 4.0 International License. The images or other third party material in this article are included in the article's Creative Commons license, unless indicated otherwise in the credit line; if the material is not included under the Creative Commons license, users will need to obtain permission from the license holder to reproduce the material. To view a copy of this license, visit http://creativecommons.org/licenses/by/4.0/ 